\title{
A New Method for Power Scheduling Management of Distributed Energy Storage System Based on Multi-Agent Structure
}

\author{
Xiaoting Shi \& Bo Yin \& Yanping Cong \& Bing Li \\ Department of Computer Science and Technology, Ocean University of China, Qingdao, China \\ Junnan Zhang \\ Shandong Taiqi Academy of New Energy Engineering Co., Ltd, Weifang, China
}

\begin{abstract}
A multi-Agent scheduling management system of distributed structure is proposed and an adaptive scheduling model which is aimed at the optimal economic benefits is established to explore more efficient methods for the scheduling problem of energy storage system. In a case that a model containing six energy storage systems provides power for a unit. It uses multi-objective optimization algorithm of three reliability factors to optimize the target. In the end, it can predict scheduling scheme of the next 24 hours. This results demonstrate the feasibility and effectiveness of the presented algorithm.
\end{abstract}

KEYWORD: Multi-Agent structure; Multi-objective optimization problem; Energy storage system scheduling

\section{INTRODUCTION}

With the development of science and technology, energy storage system scheduling is receiving more and more attention. A micro grid to a variety of uncontrollable source integrated into a manageable unit, such as solar, wind and other such uncontrollable energy. If the energy storage system is connected directly to micro grids such controllable unit for the energy storage system charging in real time, it is important for the management of energy storage system scheduling based on multi-Agent structure. Multi-Agent system scheduling must resolve the conflict and collaboration and other such task scheduling problem, what how to negotiate resource efficiency of each Agent is important to solve a certain place temporary power shortage and the monopoly of local energy storage system problems.

Currently, some domestic and foreign researchers use a multi-Agent model of distributed architecture to solve the problems of resource management task. Now, An agent is used for modeling an elementary behavior. The multi-agent system (Conte, G et al, 2015) (Filip, W et al, 1993) (Jin, Z. K et al, 1995) (Zeng, B et al, 2008) can be setup according to the specific mission or changed dynamically in response to the modification of the environment. Multi-agent structure for batch control system is proposed to make its change management easier. Four types of agents work in communicating with each other. The recipe agents instruct the recipe to equipment agents. However, the interaction between agents are achieved by means of weights. We need to continue to explore this weights. High Swallow, etc. (Gao, F. Y. 2009) proposed a negotiation mode of Contract Net (tendering-tender-awarding). It solves some problems in the area of the traditional contract net pattern generation during the past years, such as, communication blocking and communication redundancy and so on. This model not only reduces waste in the resource allocation process, but also increases the hit rate of task allocation. This is a new method of solving assignment problems. If it can set the bidding scope in the process of bidding, this mode will further improve the work efficiency. Reference (LI, R. R. 2008) uses the evaluation system, which can further reach multi-Agent communication protocols, and can immediately select qualified suppliers. However, we should explore how to choose suppliers effectively. Because of the intrinsic uncertainty of practical systems, a hybrid fuzzy Agent structure with learning and communication capabilities is first proposed (Guo, H. X et al, 2007). Reference (Dong, R.X. 2015) proposed collaboration mechanisms of hierarchical control through the Agent system of hierarchical structure, which is important to the realization of distributed structure.

This paper set up a hierarchical model on multiobjective optimization problem, raised scheduling scheme based on economic interest objectives to solve multi-objective optimization problem. This paper used the actual cost of electricity, energy storage system capacity and other data. The scheduling scheme uses three reliability factors to optimize the 
target based on 24-hour cycle optimization. The model can carry out the most economical temporary scheduling scheme to a certain remote areas.

\section{ENERGY STORAGE SCHEDULING DESIGN}

\subsection{The overall design of distributed energy storage Scheduling}

This article uses distributed structure. Unit Agent implements the real-time optimal charging and discharging strategy according to an algorithm defined by the database rules. Central control layer Agent schedules unit Agent based on the content of the task. Unit Agent gives feedback information to central control layer Agent, this can solve temporary power needs or Unit Agent has not monopoly power in that local market. In the distributed architecture, all Agent can be updated in real-time database. It not only unifies information and but also solves the problem of the whole system crashes. System structure can be found in Figure.1.

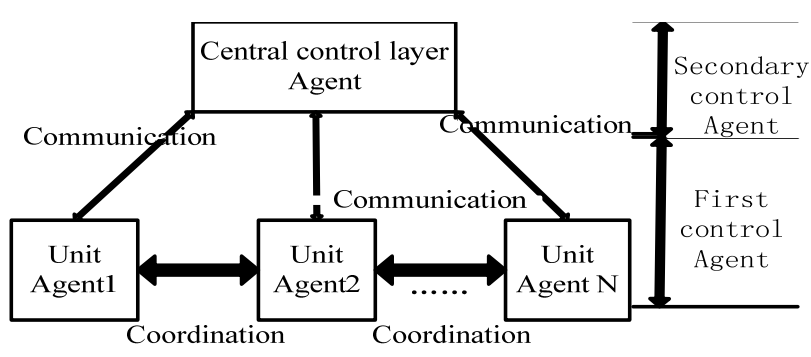

Figure 1. The overall design of distributed energy

\subsection{Agent Structural Design}

\subsubsection{First Agent Design}

1) In this paper, we use particle swarm algorithm to determine the most economical charging strategy based on parameters of the price of electricity ,the actual situation of solar and wind energy, the costs of the entire micro-grid operation and maintenance as well as energy storage capacity of the system.

2) Unit Agent assigns the right of purchase to the consumers and determines the trading order by the market according to game algorithm.

3) Unit Agent communicates with the central control layer Agent the real-time reflection of their own energy state, and the argument was submitted to the central control layer Agent.

4) Unit Agent performs the appropriate action according to the requirements of the central control layer Agent.

\subsubsection{Secondary Agent Design}

Central control layer Agent coordinates and manages all unit Agent based on the specific mission requirements. Agent registry mainly includes name, address, status parameter and so on. Central control layer has four types of Agents.

1) TXJS Agent: XJS Agent is used to transmit information between Agents, and monitors state parameters of unit Agent.

2) SF Agent: SF Agent is used to deal with information which TXJS Agent collects and makes the appropriate program.

3) EF Agent: EF Agent has a very good analysis of historical data and constantly optimizes the predictive power.

\section{CONTROL STRATEGY}

When certain place need temporary emergency power, the central control layer Agent and unit Agent will collect some of the status parameters through its own algorithms to make specific scheduling task.

Specific scheduling process as follows:

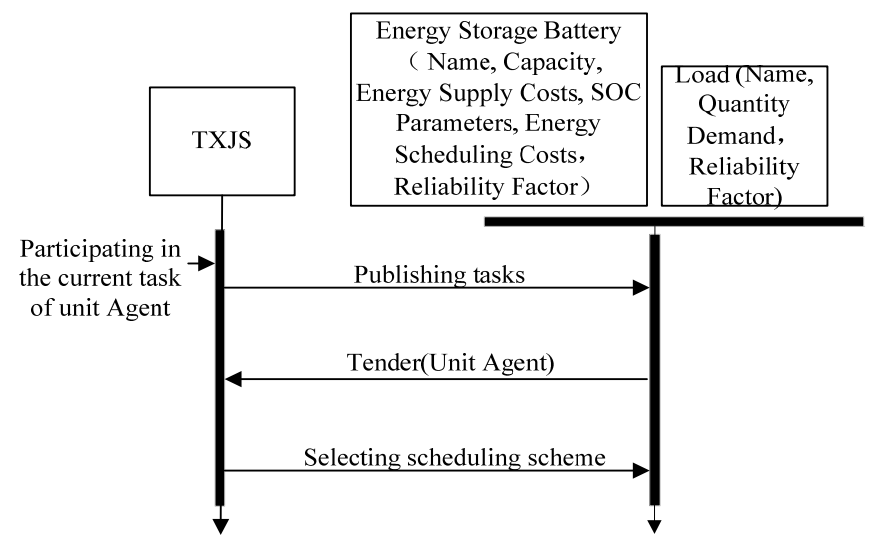

Figure 2. Secondary control Agent scheduling

Firstly, communication module of the central control layer Agent publishes this task, including the region's name, location and power demand etc. these parameters. Secondly, all unit Agent collect this information through their own monitoring module, and then give feedback to the central control layer Agent, such as storage capacity, electricity costs and so on, which details are as follows:

(1)Energy Storage Battery: Name, Energy Storage System Capacity, Energy Supply Costs, SOC Parameters, Energy Scheduling Costs, Energy Supply Reliability Factor.

(2)Load: Name, Quantity of Demand, Location, Reliability Factor.

The central control Agent begins to analyze the estimated total load demand in the 24 hours based on the parameters which are collected, and then selects the scheduling scheme.

(1)Operation and maintenance costs: 
$f_{1}=\min \operatorname{COST}_{1}$

$\operatorname{COST}_{1}=\sum_{h=1}^{24}\left(C_{M, j}+C_{\text {Price, } j}{ }^{*} S_{\text {battery }}\right)$

Where $\mathrm{j}=$ the $\mathrm{j}$-th energy storage system of the system; $\mathrm{CM}_{\mathrm{M}, \mathrm{j}}=$ operation and maintenance costs of the $\mathrm{j}$-th energy storage system of the system; CPrice, $=$ the timeshare price of the $\mathrm{j}$-th energy storage system of the system; $S_{\text {battery }}=$ power of energy storage system.

(2) Capacity of storage battery:

$f_{2}=P_{j(t)}$

$P_{S O C j, \min } \leq P_{S O C j} \leq P_{S O C j, \max }$

Where $\mathrm{P}_{j(t)}=$ output power of the $\mathrm{j}$-th energy storage system in the scheduling process; Psocj,min, $P_{\text {socj, } \max }=$ minimum and maximum output limit of the j-th energy storage system.

(3)Dispatch costs:

$f_{3}=\min \operatorname{COST}_{2}$

$\operatorname{COST}_{2}=\sum_{h=1}^{24} C_{\text {Price } 2, j}{ }^{*} M_{j}$

Where $\mathrm{C}_{\text {Price } 2, \mathrm{j}}=$ transportation costs of the $\mathrm{j}$-th energy storage system; $\mathrm{M}_{\mathrm{j}}=\mathrm{a}$ dispatch from the $\mathrm{j}$-th energy storage system.

Central control layer Agent through three reliability factors to evaluate the information reported for each energy storage system.

$w=a f_{1}+b f_{2}+c f_{3} ;(a+b+c=1)$

Then central control layer Agent selects scheduling scheme based on the value of energy storage. Three reliability factors corresponding to the costs can be changed manually. For example, when a energy storage system can not provide electricity demand or voltage is less than a predetermined voltage, b will be smaller; while some of the energy storage system provide power more economical cost, a becomes large. Algorithm process is shown in figure 3.

\section{SIMULATIONS}

This article uses MATLAB software to simulate. To verify the effectiveness of algorithm, it is tested by the following complicate example. Central control layer Agent selects scheduling scheme from the nearby six energy storage systems to provide power for a certain area. And the unfixed parameters related to the specific problem are shown in the Table 1.

The results can be drawn from the above, the central control layer Agent through a, b, c of these three reliability factors to evaluate the information reported from each energy storage system. As shown in figure 4 and 5, the whole energy storage system can predict scheduling scheme of the next 24 hours to provide power for a certain area.

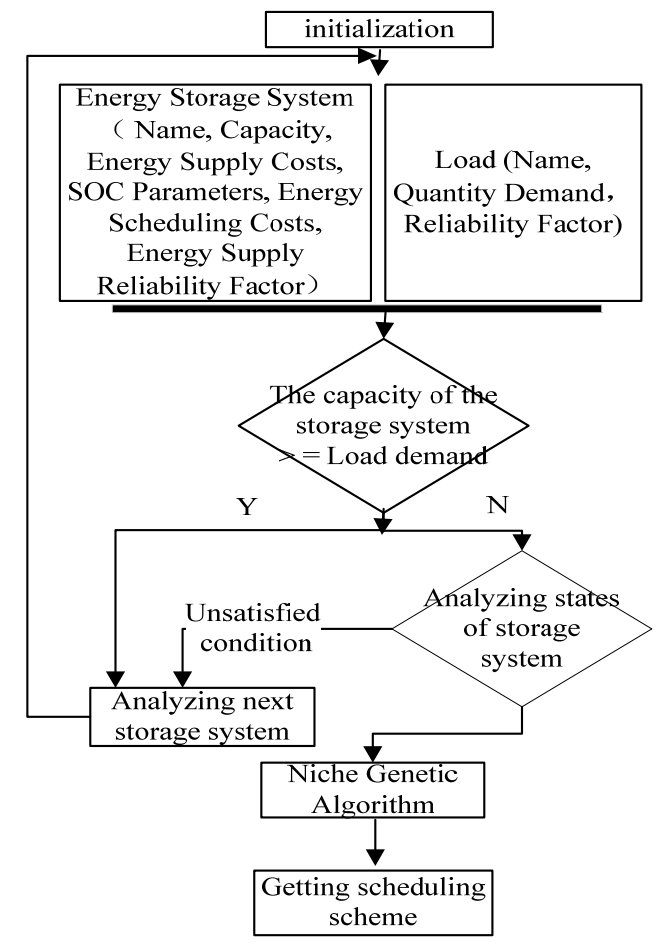

Figure 3. Block diagram of selecting scheduling scheme

Table 1. Parameters for energy storage system.

\begin{tabular}{|c|c|c|c|}
\hline \multirow[t]{2}{*}{$\begin{array}{l}\text { Energy storage } \\
\text { system }\end{array}$} & $\begin{array}{l}\text { Residential } \\
\text { electricity }\end{array}$ & $\begin{array}{l}\text { Commercial } \\
\text { electricity }\end{array}$ & Mileage \\
\hline & Yuan / kWh & Yuan / kWh & kilometer \\
\hline 1 & 0.520 & 0.843 & 100 \\
\hline 2 & 0.510 & 0.828 & 95 \\
\hline 3 & 0.510 & 0.813 & 150 \\
\hline 4 & 0.514 & 0.798 & 90 \\
\hline 5 & 0.512 & 0.800 & 130 \\
\hline 6 & 0.502 & 0.780 & 80 \\
\hline
\end{tabular}

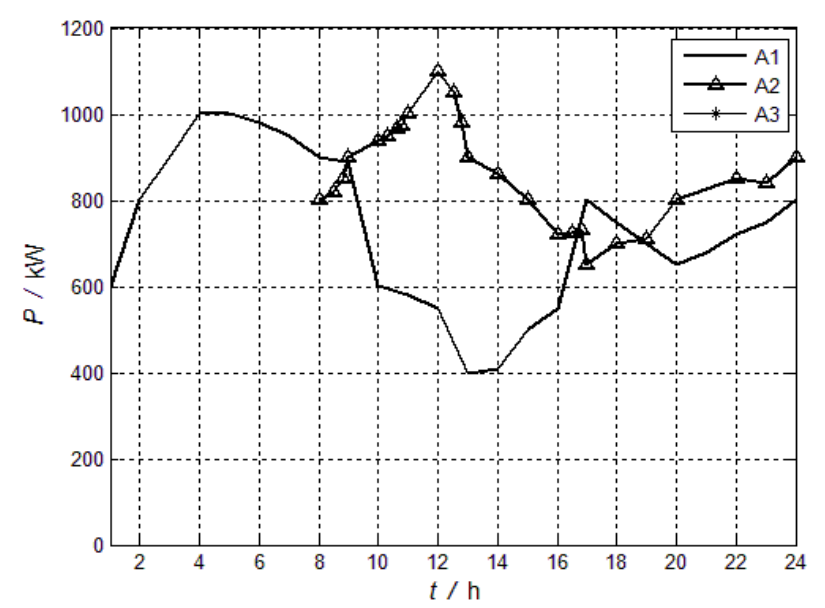

Figure 4. Energy supply A1, A2, A3 for load in 24 hours 


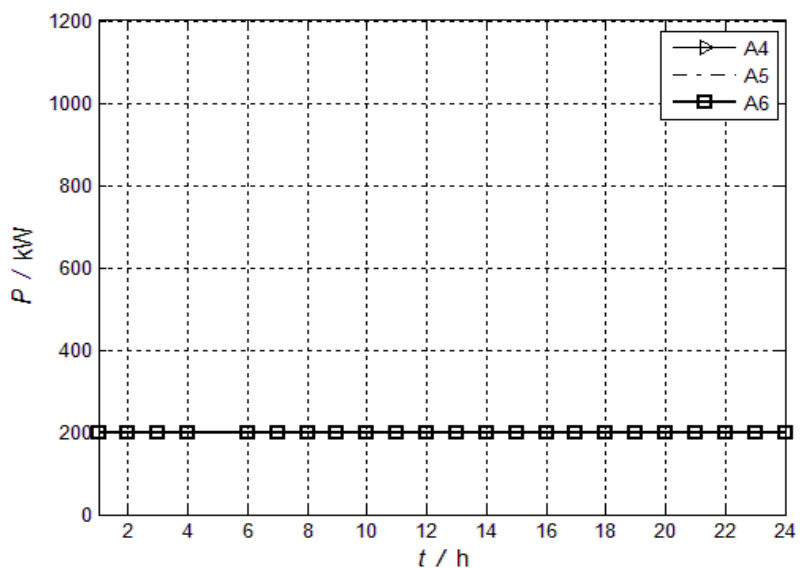

Figure 5. Energy supply A4, A5, A6 for load in 24 hours

From the figure 5 we can clearly see, 6 energy storage system is better than the others. Because the sixth energy storage system is relatively close to the load, reliability factor c is relatively large. However, due to limited capacity of storage system 6(Reliability factor $b$ is relatively small), number 6 can only provide local needs. From the simulation, the storage system 6 through its own micro-grid is its real-time charging. In figure 4 , it can be seen that so, dispatch costs of the first energy storage system is economical in addition to the sixth energy storage system. However, with the scheduling process, the operating costs rise gradually. The reliability factors of the central control layer Agent change the weighting factor of the system by manual way. The next relatively economical storage systems fill the remaining needs. From the figure we can see that the comprehensive evaluation of energy storage system $3,4,5$ is relatively $\operatorname{low}(\mathrm{a}=\mathrm{b}=\mathrm{c}=0)$. Energy storage system 3,4,5 is not scheduled in the situation of other energy storage systems to meet the load requirements.

\section{CONCLUSION}

For the energy storage scheduling problem, we put forward an improved algorithm based on three reliability factors. Used distributed structure strategy, information is shared based on first control Agent and secondary control Agent at the same time, then scheduling scheme is designed. Ensured the diversity of solution and meet the multi-objective flexible scheduling. Simulation results prove the feasibility of the algorithm. The algorithm provides a better energy scheduling abilities. The future work is to debug the three reliability factors to improve the algorithm.

\section{ACKNOWLEDGEMENTS}

This work was financially supported by national 863 plan project (2014AA052303) and strategic emerging industry in Qingdao development project (13-4$1-15-\mathrm{HY})$.

\section{REFERENCES}

Conte, G \& S, David. 2015. ROS multi-agent structure for autonomous surface vehicles. Conference article (CA). 2015 IEEE.

Dong, R.X. 2015. Research on Coordinated Operation Technique of Microgrids and Distribution Based on Hiarchical Control.2015, TM732.

Filip, W \& K. Gautam. 1993. NetView in a heterogeneous environment: Implementation of an experimental agent structure. Proceedings of the IFIP TC6/WG6.6 3rd International Symposium on Integrated Network Management. IEEE: 505-517.

Gao, F. Y. Research on the Multi-Agent Task Allocation Mechanism Based on Extended Contract Net. 2009 IEEE.

Guo, H. X \& J.Wu. 2007. Hybrid fuzzy agent structure. Journal article: $129-132+137$.

Jin, Z. K \& P. Gilbert. 1995. Multi-agent structure system for the navigation of a mobile robot. Proceedings of the 1995 IEEE 21st International Conference on Industrial Electronics, Control, and Instrumentation. Part 1 (of 2): 1130-1135.

LI, R. R. 2008. Supplier Selection System Bassed on MultiAgent. Computer Engineering, 1000-342(2008)15-0280-03.

Zeng, B \& T. H. 2008. An approach to constructing evolutionary agent structure for workflow management system based on simulation. 2008 IEEE Congress on Evolutionary Computation, CEC 2008:600-606. 